\title{
GA based Ensemble Classifier for Efficient Visual Content Information Retrieval
}

\author{
K. Vijayan \\ Research Scholar \\ Department of Computer Science \\ Manonmaniam Sundaranar University
}

\author{
C. Chandrasekar, PhD \\ Professor \\ Department of Computer Science \\ Periyar University
}

\begin{abstract}
Visual Content based Information retrieval has been one of the demanding research areas in the field of image and video retrieval. Few research works have been developed for video retrieval with aid of classification techniques. But, performance of conventional visual content based video retrieval methods was not efficient. A Gene Based Similarity Threshold Classifier (GSTC) Technique is proposed in order to improve the performance of visual content based video retrieval with higher precision, recall, F1 Measure and minimum time complexity. The GSTC Technique used Jaccard similarity coefficient to find out relevant videos in a given dataset based on query video clip. After identifying the relevant videos, GSTC Technique applied similarity threshold classifier in order to classify the videos into a different class based on diverse similarity threshold value with improved classification accuracy. Finally, GSTC Technique used genetic algorithm in order to discover the optimal similarity threshold value in population with aid of measured fitness function. This in turns, more similar related to query video are obtained for efficient video retrieval. The GSTC Technique conducts the experimental works on metrics such as classification accuracy, time complexity, F-measure, Precision and recall using three datasets.
\end{abstract}

\section{Keywords}

Genetic Algorithm, Jaccard Similarity Coefficient, Query Video, Similarity Threshold Classifier, Similarity Threshold Value, Visual Content, Videos

\section{INTRODUCTION}

Visual Content based Information Retrieval system retrieves user's required video clips from video database using their visual features for example color, texture and shape etc. Recently, many research works have been designed for visual content based video retrieval. However, performance of existing work is not effectual. Therefore, there is a requirement for a novel visual content based video retrieval technique.

Historgram Clustering Technique was designed in [1] for fast retrieval of video data. But, classification accuracy was not sufficient. An automatic setting similarity threshold (ASTS) was developed in [2] for automatically compute similarity thresholds in content-based visual information retrieval and thereby improving video retrieval performance. But, F1 measure of video retrieval was poor.

A unified approach was designed in [3] for performing content-based indexing and retrieval of digital videos from television archives with higher recall, precision and F1 measure values. However, time complexity of retrieval process was more. An Image Classification approach was intended in [4] using content based image retrieval system to classify image regions depends on their semantics. But, classification performance of was not effectual.

A survey of different techniques designed for content based video retrieval systems was analyzed in [5] for addressing the issues of video retrieval using visual information. A video retrieval system was intended in [6] to accomplish a video retrieval of a required object. This system improves precision of video retrieval. But, recall and time complexity remained unsolved.

A query-adaptive Multiple Instance Learning (q-MIL) algorithm was employed in [7] in order to retrieve the relevant frames with higher true positive rate. However, the amount of time taken for video retrieval was more. A Content Based Lecture Video Retrieval was presented in [8] by using speech and video text information for video indexing and retrieval form large lecture video archives. But, the performance of video retrieval was not effectual.

An Incremental probabilistic Latent Semantic Analysis (IpLSA) was intended in [9] to improve the performance of video retrieval. However, F-measure, Precision and recall was poor. The multi-modal spectral clustering and multi-modal ranking algorithm was designed in [10] to combine two heterogeneous features for video clustering and retrieval. But, execution time was more.

In order to overcome the existing issues, GSTC Technique is introduced. The major contribution of GSTC Technique is formulated as,

- To enhance the performance of video retrieval with higher precision, recall, F1 measure, GSTC Technique is designed.

- The Jaccard similarity coefficient is used to find the related videos based on their visual features such as shape, color, texture.

- To increase the classification performance of video retrieval with higher accuracy, similarity threshold classifier is applied in GSTC Technique.

- Further, genetic algorithm is used in GSTC Technique to achieve higher video retrieval performance.

The rest of the paper is organized as follows: In Section 2, the proposed GSTC Technique is described with aid of architecture diagram. In Section 3, Experimental settings are presented and the analysis of results is explained in Section 4. Section 5 introduces the background and reviews the related works. Section 6 provides the conclusion. 


\section{GENE BASED SIMILARITY THRESHOLD CLASSIFIER (GSTC) TECHNIQUE}

The input video clip splits into number of video frames to find their visual features in video frames and to efficiently retrieving more similar videos related to input query video clip. The GSTC Technique used similarity threshold classifier with objective of improving the classification accuracy of video retrieval. It defines different similarity threshold values during classification process based on that the videos in a given dataset are efficiently classified into similar and dissimilar video class for effectual video retrieval. Besides, GSTC Technique applied genetic optimization algorithm with objective of finding the optimal threshold value in video search for retrieving exact videos relevant to input video query with minimal time. As a result, GSTC Technique improves the precision and recall of video retrieval process with reduced time complexity. The overall architecture diagram of GSTC Technique is shown in below Figure 1.

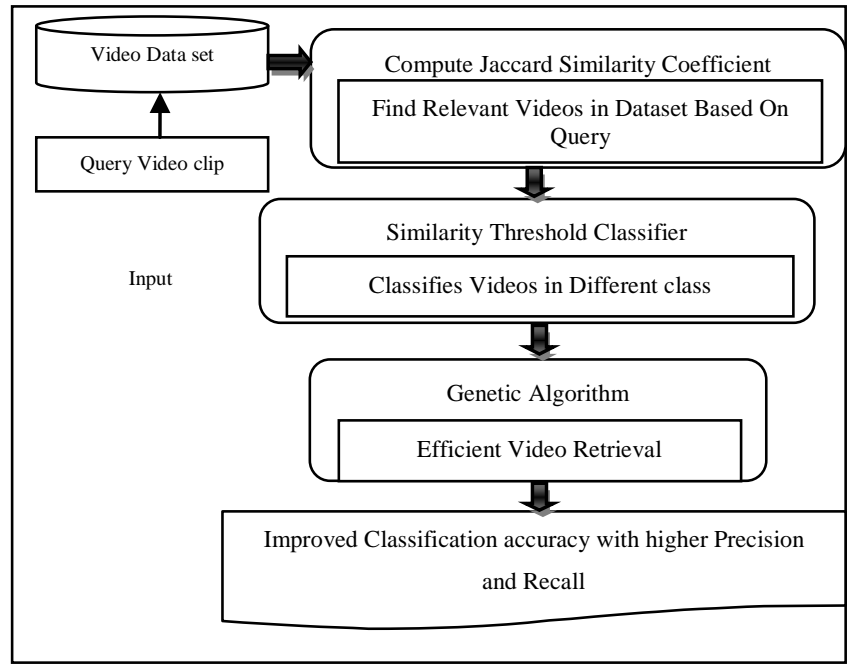

Figure 1 Architecture of GSTC Technique

As shown in Figure 1, the GSTC Technique determines Jaccard Similarity Coefficient between the input query video and videos in a given dataset. Based on the measured Jaccard similarity values, the relevant videos are efficiently identified. Next, GSTC Technique applied similarity threshold classifier with aiming at classifying the discovered relevant videos into a diverse class with higher classification accuracy. Finally, GSTC Technique employed genetic algorithm to discover the best similarity value which returns the class of more similar videos associated with the query video clip. This in turns, precision and recall are improved.

\subsection{Jaccard Similarity Coefficient}

The Jaccard Similarity Coefficient is measured with aim of improving the classification accuracy of visual content information retrieval for video mining. The input query video clip is initially divided into number of video frames and then Jaccard similar coefficient is measured based on visual features such as shape, color, texture etc. The mathematical formula for Jaccard Similarity Coefficient of video is given below,

$$
\operatorname{Sim}_{i j}=\frac{n_{i j}}{n_{i}+n_{j-n_{i j}}}
$$

From equation (1) $n_{i}$ represent number of visual features which appear in video $i$ whereas $n_{j}$ denotes the number of visual features which appear in video $j$. Here, $n_{i j}$ indicates the number of visual features which appear in both (i.e. input video query and video in a data set) video frames $i$ and $j$. The Jaccard Similarity values in the range of 0 to 1.0. Thus, video with jaccard similarity value between 0 and 1.0 is considered as similar videos to input video query (i.e. relevant videos). The remaining videos are considered as irrelevant videos.

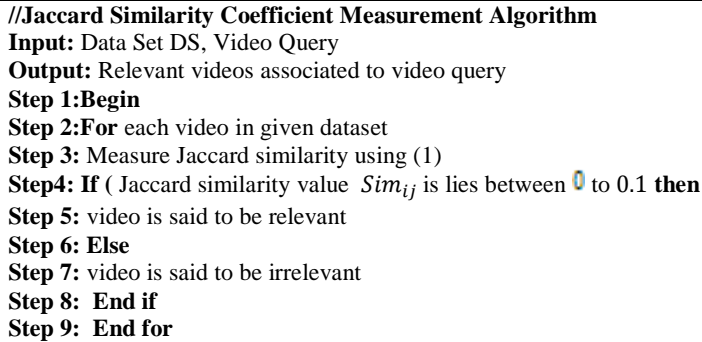

By using algorithm 1, GSTC Technique identifies relevant videos related to input video query. After computing the Jaccard similarity between videos, similarity threshold classifier is used in order to enhance the efficiency of visual content information retrieval in video mining.

\subsection{Similarity Threshold Classifier}

In Similarity Threshold Classifier, video classification based different similarity threshold values $T_{0}, . ., T_{i-1}, T_{i}, T_{i+1}, . ., T_{n}$. The group of similar videos contains objects with a degree of similarity greater than the similarity threshold value $T_{i}$ and the dissimilar videos contain the remaining ones.

The Similarity Threshold Classifier initially takes relevant videos as input. Then, classification of video as similar and dissimilar is performed based on varied similarity threshold $T_{i}$ for efficient video retrieval. This helps for improving the classification accuracy in an effective manner. To achieve better recall of video retrieval, a low similarity threshold value is initially considered.

// Similarity Threshold Classifier Algorithm
Input: Query Video, Similarity Threshold $T=0$
Output:Different class of videos related with Similarity Threshold $T$
Step 1:Begin
Step 2: For Relevant Videos in dataset
Step 3: Repeat
Step 4: Generate new Similarity Threshold value $T$
Step 5: Classify videos in a given data set into similar video and dissimilar video
with respect to generated new similarity threshold
Step 6: Until a stop criterion is met
Step 7:Return diverse class of similar videos associated with Similarity
Threshold value
Step 9: End for
Step 10:End

Algorithm 2 Similarity Threshold Classifier Algorithm

As shown in Algorithm 2, by Similarity Threshold Classifier, Similarity Threshold value $T$ is initialized considered as 0 . During the video classification process, similarity threshold value $T$ is incremented by 0.01 . Based on generated new similarity threshold value, the input relevant videos are classified as similar and dissimilar. This process repeated until the similarity threshold value $T$ reaches the maximum value of 1.0. As a result, GSTC Technique increases the classification accuracy for video retrieval.

\subsection{Genetic Algorithm}

After the classification of videos according to different 
similarity threshold, Genetic Algorithm is used in order to retrieve the more similar videos related to query video from the video database. Genetic Algorithm is an adaptive heuristic search algorithm depends on idea of natural selection and genetics. Besides, Genetic Algorithm is used to solve the optimization problem of video retrieval.The genetic algorithm discovers optimal similarity threshold values in population based on determined fitness value through performing selection, crossover, and mutation operation.

In Genetic Algorithm, fitness value is calculated based on F1 measure of video retrieval. This metric is defined in terms of precision $(\mathrm{P})$ and recall $(\mathrm{R})$. Therefore, $\mathrm{F} 1$ measure is defined as the harmonic mean of precision and recall which is mathematically formulated as,

$$
\text { Fitness }=F 1 \text { measure }=\frac{2 \times P \times R}{P+R}
$$

From equation (2), $P$ and $R$ represents the precision and recall of video retrieval. During fitness calculation, the genetic algorithm calculates the F1 values associated with different values of similarity threshold with objective of finding the best similarity threshold in video searches. The similarity threshold value was increment from zero to one by 0.01 . The genetic algorithm discovers which similarity threshold leads to higher F1 (i.e. optimal $T$ ) for a given search using a similarity function for retrieving the more similar videos.

In genetic algorithm, each similarity threshold is considered as a gene. The chromosome contains the genetic information.

\subsubsection{Initialization}

The genetic algorithm randomly initializes the gene population with help of different similarity threshold value generated in Similarity Threshold Classifier.

\subsubsection{Selection Operation}

In genetic algorithm, Selection process selects the similarity threshold from the initial population. During the selection process, similarity threshold with higher fitness values is selected.

\subsubsection{Crossover Operation}

A crossover operation is employed in genetic algorithm to change chromosomes from one generation to the next generation. The genetic algorithm applied in GSTC Technique used two Point Crossover to obtain more than one parent chromosomes and generating child chromosomes from them.

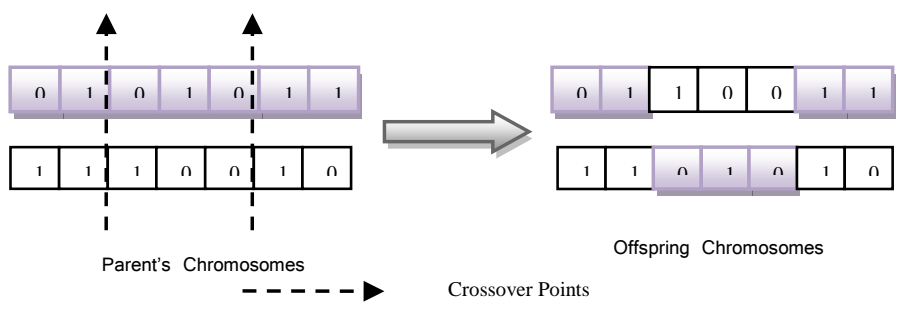

Figure 2 Process of Two Point Crossover

As shown in figure 2, through two-point crossover, two crossover points are elected and then contents between these points are swapped among two parents.

\subsubsection{Mutation Operation}

The mutation operation in genetic algorithm randomly changes the value of each bit of the chromosome along with the probability. It is called as mutation probability. In general, the probability has a very low value. Mutation process controls the genetic diversity from one generation of chromosomes to the new generation.

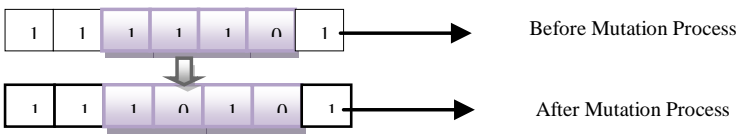

\section{Figure 3 Process of Mutation operation}

Figure 3 illustrates the mutation operation process of genetic algorithm in which the offspring chromosome value of string ' 1 ,

' is randomly changed with the chromosome string of ' 0 '. Mutation is the process of interchanging the bit for formulating the new offspring to get the optimal similarity value for video retrieval.

\section{// Genetic Algorithm based Video Retrieval}

Input: Different similarity threshold value

Output: Identify best similarity value with higher F1 value for video retrieval Step 1: Initialize the population with $n$ similarity threshold value

Step 2: For each similarity threshold value $T$ in population

Step 3: Determine fitness function using (2)

Step 4:If(fitness function of current similarity threshold value is best) then Step 5: Similarity threshold value taken as optimal $T$ to retrieve video form dataset

Step 6: Else

Step 7: Perform selection, crossover, mutation operation

Step 8: go to step 3

Step 9: End if

Step 10:End for

\section{Algorithm 3 Genetic Algorithm based Video Retrieval}

As shown in algorithm 3, genetic algorithm initializes population with $n$ similarity threshold values and computes the fitness function based on F1 measure. If fitness function of current similarity threshold value is best, then the iteration process is stopped. Then, discovered similarity threshold value considered as optimal $T$ during the video search in order to retrieve the more similar videos .Otherwise, genetic algorithm carried outs selection, crossover and mutation operation to identify an optimal similarity threshold value for video retrieval. This process is repeated until an optimal solution is attained which improves precision and recall of video retrieval with minimal time complexity.

\section{EXPERIMENTAL SETTING}

In order to analyze the performance of proposed, GSTC Technique is implemented in Java Language using three data sets namely VIRAT Video Dataset [21] and UCF Sports Action Data Set [22] and INRIA Holidays dataset [23]. The VIRAT Video Dataset consists of many videos recorded from total 11 scenes, captured through HD cameras (1080p or 720p). Besides, UCF Sports Action Data Set includes numerous videos with a collection of actions gathered from various sports and a total of 150 sequences with the resolution of 720 x 480 . Further, INRIA Holidays dataset comprises of 500 image groups in which each image group signifies a different scene or object. For conducting the experimental work, GSTC Technique takes 100 videos and 100 images from three datasets. The experimental of GSTC Technique is carried out for many instances with different number of videos and images and averagely 10 results are shown in performance analysis.

The effectiveness of GSTC Technique is compared against with two existing methods namely Histogram Clustering Technique [1] and automatic setting similarity threshold (ASTS) [2]. The performance of GSTC Technique is measured in terms of classification accuracy, time complexity, precision, recall and F1 measure. 


\subsection{Impact of Classification Accuracy}

The Classification Accuracy (CA) defined as the ratio of number of correctly classified videos to the total number of videos taken as input. It is mathematically expressed as,

$$
C A=\frac{\text { no of correctly classified videos based on query video }}{\text { total number of videos }} * 100
$$

From equation (3), CA of videos is determined with respect to different number of input videos. When the CA is higher, the method is said to be more efficient.

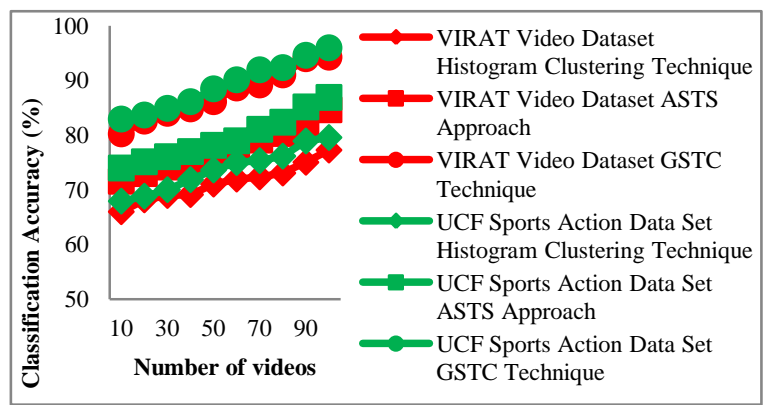

Figure 4 a) Measurement of Classification Accuracy for Video Retrieval

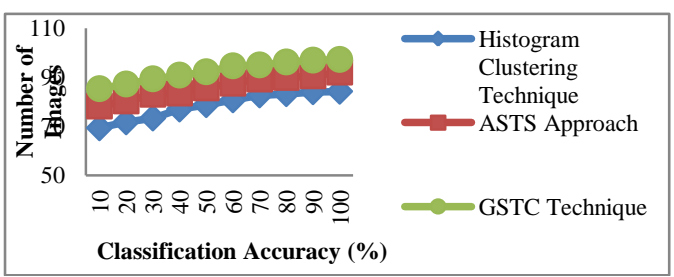

\section{Figure 4 b) Measurement of Classification Accuracy for Image Retrieval}

Figure 4 a) and b) demonstrates the impact of classification accuracy for video and image retrieval using three methods. As shown in figure, proposed GSTC Technique provides better classification accuracy for both video and image retrieval as compared to existing [1] and [2]. This is because of application of similarity threshold classifier algorithm in GSTC Technique in which similarity threshold value $T$ is dynamically incremented by 0.01 during the video classification process. The similarity threshold classifier classifies the input relevant videos into a similar and dissimilar video class with aid of generated new similarity threshold value. This is process repetitive until the similarity threshold value $T$ attains the maximum value of 1.0. This helps for improving the classification accuracy of both video and image retrieval in an effective manner. Thus, proposed GSTC Technique increases the classification accuracy of video retrieval using VIRAT Video Dataset by $23 \%$ and $13 \%$ when compared to [1] and [2] respectively. Besides, proposed GSTC Technique enhances the classification accuracy of video retrieval using UCF Sports Action Data Set by $21 \%$ and $12 \%$ when compared to [1] and [2] respectively. Furthermore, proposed GSTC Technique improves the classification accuracy of image retrieval using INRIA Holidays dataset by $18 \%$ and $8 \%$ when compared to [1] and [2] respectively.

\subsection{Impact of Time Complexity}

The Time Complexity (TC) measures the amount of time taken for retrieving more similar videos form a given a dataset. It is mathematically represented as follows,
$T C=N *$ Time $($ Retrieving one video $)$

From equation (4), the amount of time required for video retrieval is measured in which $N$ denotes the total number of videos. When the TC is lower, the method is said to be more effective.

Table 1 a) Tabulation of Time Complexity for Video Retrieval

\begin{tabular}{|c|c|c|c|c|c|c|}
\hline \multirow{2}{*}{$\begin{array}{c}\text { No. of } \\
\text { videos }\end{array}$} & \multicolumn{6}{|c|}{ Time Complexity (ms) } \\
\cline { 2 - 7 } & \multicolumn{2}{|c|}{ VIRAT Video Dataset } & \multicolumn{2}{c|}{ UCF Sports Action Data Set } \\
\cline { 2 - 7 } & $\begin{array}{c}\text { Histogram } \\
\text { Clustering } \\
\text { Technique }\end{array}$ & $\begin{array}{c}\text { AST } \\
\text { S }\end{array}$ & $\begin{array}{c}\text { GST } \\
\text { C }\end{array}$ & $\begin{array}{c}\text { Histogram } \\
\text { Clustering } \\
\text { Technique }\end{array}$ & ASTS & GSTC \\
\hline 10 & 23.2 & 21.9 & 15.7 & 21.3 & 19.5 & 14.2 \\
\hline 20 & 30.4 & 27.1 & 19.3 & 28.8 & 25.2 & 18.5 \\
\hline 30 & 36.9 & 31.8 & 23.6 & 35.6 & 29.7 & 21.8 \\
\hline 40 & 43.8 & 34.2 & 26.2 & 42.3 & 32.9 & 22.7 \\
\hline 50 & 52.1 & 37.6 & 29.1 & 50.1 & 35.5 & 26.9 \\
\hline 60 & 53.9 & 43.8 & 33.7 & 52.8 & 40.6 & 30.3 \\
\hline 70 & 57.2 & 45.7 & 38.1 & 55.7 & 43.4 & 35.1 \\
\hline 80 & 60.8 & 49.6 & 41.0 & 59.1 & 46.2 & 37.8 \\
\hline 90 & 64.3 & 52.1 & 42.8 & 62.8 & 50.9 & 39.2 \\
\hline 100 & 67.5 & 57.9 & 44.9 & 65.2 & 56.7 & 43.7 \\
\hline
\end{tabular}

Table 1 b) Tabulation of Time Complexity for Image Retrieval

\begin{tabular}{|c|c|c|c|}
\hline \multirow{2}{*}{$\begin{array}{c}\text { Number } \\
\text { of Images }\end{array}$} & \multicolumn{3}{|c|}{ Time Complexity (ms) } \\
\cline { 2 - 4 } & $\begin{array}{c}\text { Histogram } \\
\text { Clustering } \\
\text { Technique }\end{array}$ & $\begin{array}{c}\text { ASTS } \\
\text { Approach }\end{array}$ & $\begin{array}{c}\text { GSTC } \\
\text { Technique }\end{array}$ \\
\hline 10 & 18.7 & 14.9 & 9.5 \\
\hline 20 & 20.5 & 16.3 & 11.6 \\
\hline 30 & 21.6 & 17.5 & 13.5 \\
\hline 40 & 24.3 & 20.7 & 16.8 \\
\hline 50 & 29.5 & 23.8 & 17.9 \\
\hline 60 & 32.1 & 27.1 & 20.3 \\
\hline 70 & 35.6 & 31.5 & 22.5 \\
\hline 80 & 40.8 & 34.9 & 25.6 \\
\hline 90 & 42.4 & 38.4 & 28.7 \\
\hline 100 & 45.7 & 40.6 & 31.2 \\
\hline
\end{tabular}

Table 1 a) and b) portrays the comparative result analysis of time complexity for both video and image retrieval using three methods based on diverse number of videos and images taken in the range of 10-100. From the table value, it is clear that the time complexity using proposed GSTC Technique is lower for the both video and image retrieval when compared to existing [1] and [2] respectively.

This is owing to use of genetic algorithm in GSTC Technique. With aid of this algorithmic process, GSTC Technique significantly selects optimal similarity threshold value based on fitness function calculation during video search in order to improve the video retrieval performance with lower time. This assists for reducing the time complexity of both video and image retrieval in an effectual manner. Therefore, GSTC Technique lessens the amount time taken for video retrieval using VIRAT Video Dataset by $36 \%$ and $23 \%$ when compared to [1] and [2] respectively. In addition, proposed 
GSTC Technique minimizes the time complexity of video retrieval using UCF Sports Action Data Set by $39 \%$ and 24\% when compared to [1] and [2] respectively. Furthermore, proposed GSTC Technique lessens the time complexity of image retrieval using INRIA Holidays dataset by $38 \%$ and $26 \%$ when compared to [1] and [2] respectively.

\subsection{Impact of Precision}

The precision measures the ratio of number of similar videos retrieved based on optimal threshold value to total number of videos taken as input. It is mathematically formulated as,

Precision $(P)=\frac{\text { Number of similar videos retrieved }}{\text { Total number of videos }} * 100$

From equation (5), the precision rate of video retrieval is measured. When the precision value is higher, the method is said to be more efficient.

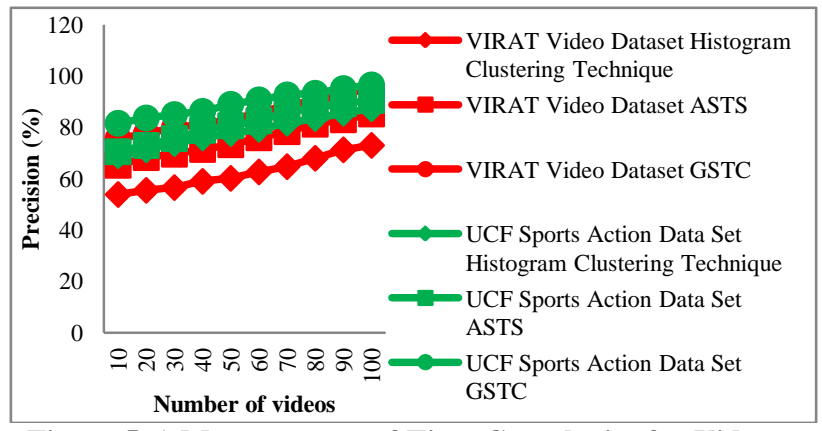

Figure 5 a) Measurement of Time Complexity for Video Retrieval

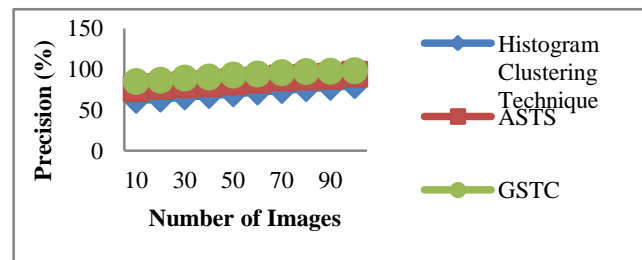

Figure 5 b) Measurement of Time Complexity for Image Retrieval

Figure 5 a) and b) illustrates the impact of precision for video and image retrieval using three methods. As revealed in figure, proposed GSTC Technique provides better precision for both video and image retrieval as compared to existing [1] and [2]. This is due to application of genetic algorithm in GSTC Technique in which fitness function is calculated for each similarity threshold value in gene population based on F1 measures of video retrieval. This helps for GSTC Technique to identify the best similarity threshold value for efficiently retrieving similar videos related input query video. This in turn helps for enhancing the precision of both video and image retrieval in a significant manner. As a result, GSTC Technique improves the precision of video retrieval using VIRAT Video Dataset by $37 \%$ and $13 \%$ when compared to [1] and [2] respectively. As well, proposed GSTC Technique enhances the precision of video retrieval using UCF Sports Action Data Set by $14 \%$ and $10 \%$ when compared to [1] and [2] respectively. Furthermore, proposed GSTC Technique improves the precision of image retrieval using INRIA Holidays dataset by $31 \%$ and $9 \%$ when compared to Histogram Clustering Technique [1] and ASTS Approach [2] respectively.

\subsection{Impact $t$ of Recall}

The recall measures the ratio of number of correctly retrieved similar videos based on optimal threshold value to total number of videos taken as input. It is mathematically formulated as,

Recall $=\frac{\text { Number of correctly retrieved similar videos }}{\text { Total number of videos }} * 100$

From equation (6), the precision rate of video retrieval is measured. When the precision value is higher, the method is said to be more efficient.

Table 2 a) Tabulation of Recall for Video Retrieval

\begin{tabular}{|c|c|c|c|c|c|c|}
\hline \multirow{2}{*}{$\begin{array}{c}\text { Numbe } \\
\text { r of } \\
\text { videos }\end{array}$} & \multicolumn{6}{|c|}{ Recall (\%) } \\
\cline { 2 - 7 } & $\begin{array}{c}\text { VIRAT Video Dataset } \\
\text { Histogra } \\
\mathbf{m} \\
\text { Clustering } \\
\text { Technique }\end{array}$ & $\begin{array}{c}\text { AST } \\
\text { S }\end{array}$ & $\begin{array}{c}\text { GST } \\
\mathbf{C}\end{array}$ & $\begin{array}{c}\text { Histogra } \\
\mathbf{m} \\
\text { Clustering } \\
\text { Technique }\end{array}$ & $\begin{array}{c}\text { AST } \\
\text { S }\end{array}$ & $\begin{array}{c}\text { GST } \\
\text { C }\end{array}$ \\
\hline 10 & 45.6 & 51.21 & 63.75 & 58.19 & 60.17 & 71.39 \\
\hline 20 & 47.56 & 53.69 & 65.78 & 60.49 & 63.52 & 73.69 \\
\hline 30 & 49.65 & 55.71 & 67.13 & 62.36 & 65.89 & 75.95 \\
\hline 40 & 51.43 & 57.53 & 69.31 & 65.89 & 67.58 & 77.63 \\
\hline 50 & 53.29 & 59.89 & 72.69 & 67.54 & 69.56 & 79.41 \\
\hline 60 & 55.96 & 61.32 & 74.15 & 69.42 & 71.25 & 82.59 \\
\hline 70 & 57.63 & 64.89 & 76.98 & 71.67 & 73.54 & 85.14 \\
\hline 80 & 59.16 & 65.23 & 78.62 & 73.98 & 75.21 & 87.76 \\
\hline 90 & 60.56 & 68.96 & 81.47 & 75.69 & 77.25 & 89.86 \\
\hline 100 & 62.69 & 72.23 & 83.69 & 77.52 & 79.17 & 91.63 \\
\hline
\end{tabular}

Table 2 b) Tabulation of Recall for Image Retrieval

\begin{tabular}{|c|c|c|c|}
\hline \multirow{2}{*}{$\begin{array}{c}\text { Number } \\
\text { of Images }\end{array}$} & \multicolumn{3}{|c|}{ Recall (\%) } \\
\cline { 2 - 4 } & $\begin{array}{c}\text { Histogram } \\
\text { Clustering } \\
\text { Technique }\end{array}$ & ASTS & GSTC \\
\hline 10 & 56.89 & 61.87 & 78.52 \\
\hline 20 & 58.69 & 64.96 & 81.89 \\
\hline 30 & 61.58 & 66.45 & 83.64 \\
\hline 40 & 63.69 & 69.58 & 85.47 \\
\hline 50 & 65.96 & 72.69 & 87.34 \\
\hline 60 & 67.12 & 75.12 & 89.63 \\
\hline 70 & 69.45 & 78.37 & 91.56 \\
\hline 80 & 71.38 & 81.63 & 94.59 \\
\hline 90 & 73.69 & 83.75 & 96.28 \\
\hline 100 & 75.85 & 87.23 & 98.69 \\
\hline
\end{tabular}

Table 2 a) and b) explains the comparative result analysis of recall is obtained for both video and image retrieval using three methods with respect to varied number of videos and images taken in the range of 10-100. From the table value, it is expressive that the recall using proposed GSTC Technique is higher for the both video and image retrieval as compared to existing [1] and [2].

This is because of usage of genetic algorithm in GSTC Technique where it determines fitness function for each similarity threshold value in gene population with respect to F1 measures of video retrieval. This supports for GSTC Technique to discover the best similarity threshold value for correctly retrieving similar videos related input query video. This in turn helps for increasing the recall of both video and image retrieval in an effectual manner. Therefore, GSTC Technique improves the recall of video retrieval using VIRAT Video Dataset by $35 \%$ and $20 \%$ when compared to Histogram Clustering Technique [1] and ASTS Approach [2] respectively. In addition, proposed GSTC Technique enhances 
the recall of video retrieval using UCF Sports Action Data Set by $20 \%$ and $16 \%$ when compared to [1] and [2] respectively. Moreover, proposed GSTC Technique improves the recall of image retrieval using INRIA Holidays dataset by $34 \%$ and $20 \%$ when compared to [1] and [2] respectively.

\subsection{Impact of F1 Measure}

F1 measure described as harmonic mean of precision and recall of video retrieval process. The mathematical formula is shown in below,

$$
F 1 \text { measure }=\frac{2 \times P \times R}{P+R}
$$

From equation (7), $P$ and $R$ represents the precision and recall of video retrieval. When F1 measure value is higher, the method is said to be more effectual.

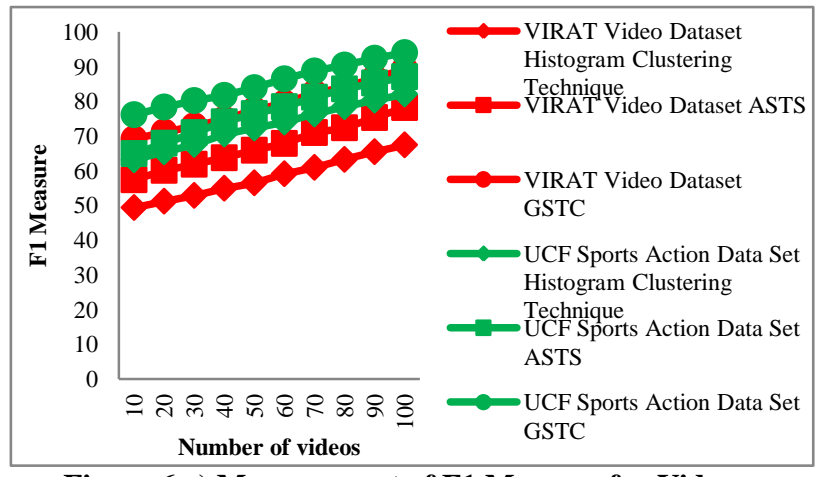

Figure 6 a) Measurement of F1 Measure for Video Retrieval

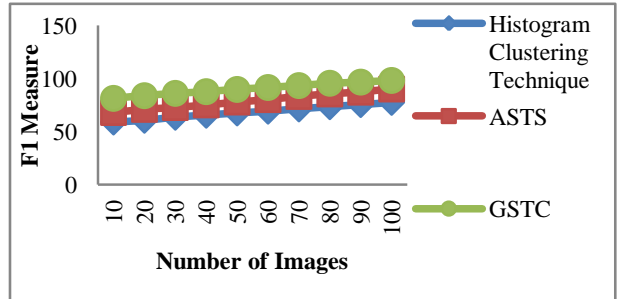

Figure 6 b) Measurement of F1 Measure for Image Retrieval

Figure 6 a) and b) reveals the impact of F1 Measure for video and image retrieval using three methods. As shown in figure, proposed GSTC Technique provides better F1 Measure results for both video and image retrieval as compared to existing [1] and [2]. This is due to application of genetic algorithm in which is fitness function of similarity threshold value in gene population is evaluated based on precision and recall rate of video retrieval during classification. Besides, genetic algorithm in GSTC Technique finds optimal similarity threshold based on fitness function. This supports for GSTC Technique to increases the F1 Measure for both video and image retrieval. Hence, proposed GSTC Technique improves the FI Measures of video retrieval using VIRAT Video Dataset by $36 \%$ and $17 \%$ when compared to [1] and [2] respectively. Further, proposed GSTC Technique enhances the FI Measures of video retrieval using UCF Sports Action Data Set by $44 \%$ and $11 \%$ when compared to [1] and [2] respectively. Besides, proposed GSTC Technique increases the FI Measures of image retrieval using INRIA Holidays dataset by $33 \%$ and 15\% when compared to [1] and [2] respectively.

\section{RELATED WORKS}

Fisher Kernel Temporal Variation-based Relevance Feedback was presented in [11] for performing video retrieval with higher precision rate. But, precision was not at required level. Correspondence-Latent Dirichlet Allocation (corr-LDA) probabilistic framework was designed in [12] for solving issues and enhancing the performance content-based video indexing and retrieval with higher accuracy. However, space complexity remained unsolved.

Content Based Video Retrieval was developed in [13] with application of cluster overlapping using dissimilar spatiotemporal features for retrieving videos from the database. However, time complexity of video retrieval was higher. A content-based video indexing and retrieval algorithm was intended in [14] with aid of key-frames texture, edge, and motion features in order to increase the performing of video indexing and retrieval. But, true positive rate of video retrieval was not considered.

2-D Correlation Algorithm was presented in [15] for enhancing the performance of content based video retrieval system with higher accuracy. But, execution time for video retrieval was more. Content based video retrieval was carried out in [16] with help of Hadamard matrix and discrete wavelet transform with objective of improving accuracy of video retrieval rate. However, the true positive rate of video retrieval was poor.

Video Retrieval performance was improved in [17] through classification of video database by using multiple Frames depends on texture information. But, precision rate of video retrieval was lower. Content based video retrieval system was presented in [18] with application of entropy based shot detection method to accomplish video indexing and retrieval. But, video retrieval performance was not sufficient.

An efficient content based video retrieval system was designed in [19] with aid of color feature for achieving effective result in video retrieval. However, retrieving similar videos was remained unsolved. A Multiple Feature Hashing was presented in [20] for large-scale near-duplicate video retrieval and address the accuracy and the scalability issues. But, time and space complexity of video retrieval was not considered.

\section{CONCLUSION}

An effective GSTC Technique is developed with objective of enhancing the performance of visual content based video retrieval. Through Jaccard similarity coefficient measurement, the relevant videos are identified. Then, similarity threshold classifier is used for classifying the videos into a dissimilar class based on varied similarity threshold value. At last, GSTC Technique applied genetic algorithm with objective of finding the best similarity threshold value in population based on calculated fitness function which in turn helps for enhancing the precision, recall, F1 measure with minimum time complexity. With the experiments conducted for GSTC Technique, it is observed that the precision and recall of video retrieval provides more accurate results with reduced time complexity as compared to state-of-the-art works.

\section{REFERENCES}

[1] D.Saravanan, Vaithyasubramanian, K.N. Jothi Vengatesh, "Video Content Retrieval Using Historgram Clustering Technique", Procedia Computer Science, Elsevier, Volume 50, Pages 560 - 565, 2015

[2] Izaquiel L. Bessas, Flávio L. C. Pádua1, Guilherme T. de Assis, Rodrigo T. N. Cardoso and Anisio Lacerda, "Automatic and online setting of similarity thresholds in content-based visual information retrieval problems", 
EURASIP Journal on Advances in Signal Processing, Springer, Volume 32, 2016

[3] Celso L. Souza, Flávio L. C. Pádua, Cristiano F. G. Nunes, Guilherme T. Assis, Giani D. Silva, "A unified approach to content-based indexing and retrieval of digital videos from television archives", Artificial Intelligence Research, Volume 3, Issue 3, Pages 49-61, 2014

[4] K. Venu Gopala Rao, P. Prem Chand, M.V. Ramana Murthy, "Image Classification Using Content Based Image Retrieval System", International Journal of Image Processing and Applications, Volume 2, Issues 1, Pages $85-91,2011$

[5] P. N Chatur, R. M. Shende, "Simple Review on Content Based Video Images Retrieval", International Journal of Engineering Research \& Technology (IJERT)", Volume 2, Issue 3, Pages 1-6, March - 2013

[6] Yuan-Hao Lai and Chuan-Kai Yang, "Video Object Retrieval by Trajectory and Appearance", IEEE Transactions on Circuits and Systems for Video Technology, Volume 25, Issue 6, Pages1027-1037, June 2015

[7] Ting-Chu Lin, Min-Chun Yang, Chia-Yin Tsai, and YuChiang Frank Wang, "Query-Adaptive Multiple Instance Learning for Video Instance Retrieval", IEEE Transactions on Image Processing, Volume 24, Issue 4, Pages 1330 - 1340, April 2015

[8] Haojin Yang and Christoph Meinel, "Content Based Lecture Video Retrieval Using Speech and Video Text Information", IEEE Transactions on Learning Technologies, Volume 7, Issue 2, Pages 143-154, AprilJune 2014

[9] Ruben Fernandez-Beltran, Filiberto Pla, "Incremental probabilistic Latent Semantic Analysis for video retrieval", Image and Vision Computing, Elsevier, Volume 38, Pages 1-12, 2015

[10] JunweiHan, XiangJi, XintaoHu, JungongHan, TianmingLiu, "Clustering and retrieval of video shots based on natural stimulus fMRI", Neurocomputing, Elsevier, Volume 144, Pages 128-137, 2014

[11] Ionut Mironic, Bogdan Ionescu, Jasper Uijling, NicuSebe, "Fisher Kernel Temporal Variation-based Relevance Feedback for video retrieval", Computer Vision and Image Understanding, Elsevier, Volume 143, Pages 38-51, 2016

[12] Rahul Radhakrishnan Iyer, Sanjeel Parekh, Vikas Mohandoss, Anush Ramsurat, Bhiksha Raj, Rita Singh, "Content-based Video Indexing and Retrieval Using
Corr-LDA", Computer Science, Information Retrieval, Pages 1-7, 2016

[13] Deepak C R , Sreehari S , Gokul M , Anuvind B, "Content Based Video Retrieval Using Cluster Overlapping", International Journal of Computational Engineering Research, Volume 03, Issue 5, Pages 104108,2013

[14] M.Ravinder and T.Venugopal, "Content-Based Video Indexing and Retrieval using Key frames Texture, Edge and Motion Features", International Journal of Current Engineering and Technology, Volume 6, Issue 2, Pages 672-676, April 2016

[15] Navdeep Kaur, Mandeep Singh, "Content Based Video Retrieval with Frequency Domain Analysis Using 2-D Correlation Algorithm", Volume 4, Issue 9, Pages 388393, September 2014

[16] Sajad Mohamadzadeh and Hassan Farsi, "Content Based Video Retrieval Based On HDWT and Sparse Representation", Image Analysis and Strereology, Volume 35, Issue 2, Pages 67-80, 2016

[17] Mohd. Aasif Ansari, Hemlata Vasishtha, "Enhanced Video Retrieval and Classification of Video Database Using Multiple Frames Based on Texture Information", International Journal of Computer Science and Information Technologies, Volume 6, Issue 2, Pages 1740-1745, 2015

[18] P. M. Kamde, Sankirti Shiravale, S. P. Algur, "Entropy Supported Video Indexing for Content based Video Retrieval", International Journal of Computer Applications, Volume 62, Issue 17, Pages 1-6, January 2013

[19] Tejaswi Potluri, Gnaneswararao Nitta, "Content Based Video Retrieval Using Dominant Color of the Truncated Blocks of Frame", Journal of Theoretical and Applied Information Technology, Volume 85, Issue 2, Pages 162171,2016

[20] Jingkuan Song, Yi Yang, Zi Huang, Heng Tao Shen, and Jiebo Luo, "Effective Multiple Feature Hashing for Large-Scale Near-Duplicate Video Retrieval", IEEE Transactions on Multimedia, Volume 15, Issues 8, Pages 1999-2008, December 2013

[21] VIRAT Video Dataset: http://www.viratdata.org/

[22] UCF Sports Action Data Set: http://crcv.ucf.edu/data/UCF_Sports_Action.php

[23] INRIA Holidays http://lear.inrialpes.fr/ jegou/data.php 